\title{
Exact Cosmological Solutions of Gravitational Theories
}

\author{
Robert T. JANTZEN \\ Villanova University, Villanova, PA 19085, USA and \\ International Center for Relativistic Astrophysics, \\ Department of Physics, University of Rome, I-00185 Rome, Italy \\ Received 22 September 1986 \\ Physics Letters B186, 290-296 (1987)
}

\begin{abstract}
A global picture is drawn tying together most exact cosmological solutions of gravitational theories in four or more spacetime dimensions.
\end{abstract}

\section{Introduction}

The search for exact solutions of gravitational theories in four spacetime dimensions has often become an end in itself, a game in which one seeks to write down closed form expressions or at least quadratures which solve some particular case of some set of equations, without worrying about interpretation, placing them into context or drawing some significant conclusion about properties of the original equations. Now this game, increasingly more difficult to play in four dimensions, is escaping into higher dimensions where there is little resistance. This article wishes to point out that almost all known exact cosmological solutions of deterministic field equations can be understood in terms of a few simple ideas. (Perfect fluids with no equation of state or imperfect fluids are meant to be excluded by the phrase deterministic field equations.) The very existence of these exact solutions and their relationship to each other depends crucially on the underlying structure of the field equations themselves, a fact which is rarely appreciated.

These remarks are confined to spatially homogeneous classical solutions of gravitational theories in which the purely gravitational variables have Einsteinlike equations, i.e., do not involve "higher derivatives". For such theories a well developed Lagrangian/Hamiltonian formulation of the field equations helps to understand the exact solutions in terms of an elegant geometrical picture. This approach, initiated by Arnowit, Deser and Misner [1] for Einstein's equations in four dimensions, was developed by Misner [2-4] as a powerful way of viewing the dynamics of spatially homogeneous cosmological models. It extends to higher 
dimensional theories in a natural way, including Brans-Dicke and supersymmetric variations of Einstein's theory coupled to various matter sources. Henneaux [5] gives the details for ten-dimensional supergravity, for example.

\section{Spatially Homogeneous Models}

By choosing the time coordinate lines orthogonal to the spatial sections, the $d$-dimensional spacetime metric can be expressed at least locally in the form [6-9]

$$
{ }^{(d)} \mathrm{g}=-N(t)^{2} d t \otimes d t+g_{a b}(t) \omega^{a} \otimes \omega^{b}
$$

on the manifold $R \times(G / H)$, where $\left\{\omega^{a}\right\}$ are 1-forms on $G / H$ dual to the frame $\left\{e_{a}\right\}_{a=1, \ldots, D}($ let $D=d-1)$ characterized by structure functions $C^{a}{ }_{b c}=$ $\omega^{a}\left(\left[e_{a}, e_{b}\right]\right)$ on $G / H$. These are constants in the simply transitive case where $H$ is the identity subgroup of the isometry group $G$ and $\left\{e_{a}\right\}$ is a left invariant frame on $G / H=G$ (i.e., a basis of the Lie algebra of $G$ ), but they are functions on the left coset space $G / H$ in the nontrivial multiply transitive case, where $H$ is a continuous subgroup. In the first case the matrix $\mathbf{g} \equiv\left(g_{a b}\right)$ is an arbitrary positive definite symmetric matrix, while in the second case it must satisfy additional linear constraints $[8,9]$. Additional spatial symmetries in the first case lead to similar constraints; such cases might be called trivially multiply transitive as opposed to those "nontrivial" models which have no subgroup acting simply transitively on the orbits.

The choice of lapse function $N(t)$ determines the parametrization of the family of homogeneous spatial sections, i.e., the time function. The proper time $\tau$, defined by $d \tau=N(t) d t=\omega^{\perp}$ corresponds to unit lapse. In expanding models with an initial singularity, $\tau$ is usually chosen so that this singularity occurs at $\tau=0$. Defining $\omega^{0}=d t$ leads to the spacetime 1-forms $\omega^{\alpha}$ dual to the frame $\left\{e_{\alpha}\right\}_{\alpha=0,1, \ldots, D}$, where $e_{0}=\partial / \partial t$ and $e_{\perp}=N^{-1} e_{0}$ is the unit normal to the spatial sections. Clearly the choice of time function should be made to simplify the equations of motion for $\mathbf{g}=\left(g_{a b}\right)$. In order to obtain exact solutions (at least up to quadratures) one must decouple the equations in some way, a problem which depends critically on the choice of time.

For the sake of brevity, consider only models which are "diagonalizable" and therefore can be assumed to be diagonal, i.e., the matrix $\mathbf{g}$ is diagonal

$$
\mathbf{g}=\mathbf{g}_{\mathrm{D}}=\operatorname{diag}\left(g_{11}, \ldots, g_{D D}\right)
$$

and hence its natural logarithm is an arbitrary diagonal matrix which can be decomposed into its pure trace and tracefree parts

$$
\begin{aligned}
& \mathbf{g}_{\mathrm{D}}=e^{2 \alpha} e^{2 \boldsymbol{\beta}}, \\
& \frac{1}{2} \ln \mathbf{g}_{\mathrm{D}}=\alpha \mathbf{1}+\beta^{A} \mathbf{e}_{A}, \\
& \operatorname{Tr} \mathbf{e}_{A}=0, \quad \operatorname{Tr} \mathbf{e}_{A} \mathbf{e}_{B}=D(D-1) \delta_{A B},
\end{aligned}
$$

where the normalization of the tracefree basis is the most natural one in view of considerations described below. Most known exact solutions fall into this 
diagonal class. The existence of diagonal solutions of the field equations depends on the symmetry type, the choice of frame and the form of the field equations (including the symmetry of the source, etc.) and is usually connected in some way with discrete or continous spacetime symmetry of some kind.

Even though the present remarks will be confined to such diagonal solutions, a similar analysis of the few exact solutions not of this form is even more crucial due to their complexity. Moreover, it is absolutely essential to rely on these ideas to appreciate the general spatially homogeneous case, which does not admit exact solution $[10,11]$.

\section{$3 \quad$ Field Equations}

Since we wish to focus on the gravitational properties of higher dimensional models, we consider the Einstein equations with all other fields lumped into the energy-momentum tensor (including a possible cosmological constant). This assumes that the gravitational part of the classical field equations can be represented in this way, thus excluding "higher derivative" theories. These equations

$$
0=\left.\left.M_{\alpha \beta} \equiv\right|^{(d)} g\right|^{1 / 2}\left({ }^{(d)} G_{\alpha \beta}-k T_{\alpha \beta}\right),\left.\left.\quad\right|^{(d)} g\right|^{1 / 2}=N g^{1 / 2}
$$

(where metric quantities without the leading superscript ' (d)' refer to the spatial metric) naturally split into evolution equations $0=M_{a b}$ which evolve the spatial metric and constraints on the solutions of those equations

$$
\begin{array}{ll}
0=\mathcal{H} \equiv 2 N^{-1} M^{\perp}{ }_{\perp} \equiv 2 M^{0}{ }_{\perp} & \text { (super-Hamiltonian constraint) } \\
0=\mathcal{H}_{a} \equiv 2 N^{-1} M^{\perp}{ }_{a} \equiv 2 M^{0}{ }_{a} & \text { (supermomentum constraint) }
\end{array}
$$

The Einstein equations may also be written in "Ricci form"

$$
0=P_{\alpha \beta} \equiv{ }^{(d)} R_{\alpha \beta}-k E_{\alpha \beta}, \quad E_{\alpha \beta} \equiv T_{\alpha \beta}-(d-2)^{-1} T_{\gamma}^{\gamma} g_{\alpha \beta},
$$

a form very convenient for static solutions in discussions of spontaneous compactification. However, for dynamical solutions a compromise is preferable, namely the Ricci evolution equations $0=P_{a b}$, supplemented by the Einstein constraints rather than more equations involving second time derivatives. For our purposes it is crucial to choose the lapse function to be an explicit function of the spatial metric given by

$$
N_{\mathrm{TTG}}=2 D(D-1) g^{1 / 2}
$$

this choice of time will be called Taub time gauge. In fact, the Ricci evolution equations are just the variational equations obtained from the usual ADM Einstein Lagrangian [12] with this explicit choice of the lapse, although for some symmetry types one must add a nonpotential force to the system to get the correct equations. For diagonal models, these equations are explicitly

$$
\left(\ln \mathbf{g}_{\mathrm{D}}\right)^{*}=-2 N_{\mathrm{TTG}}^{2}(\mathbf{R}-k \mathbf{E})
$$


where $\mathbf{R}=\left(R_{b}^{a}\right)$ is the matrix of mixed components of the spatial Ricci tensor and $\mathbf{E}=\left(E^{a}{ }_{b}\right)$.

In general, the evolution equations may be obtained from the ADM Lagrangian or its associated Hamiltonian

$$
\begin{aligned}
& L_{\mathrm{ADM}}=T-U, \quad H_{\mathrm{ADM}}=T+U=N \mathcal{H}, \\
& T=(4 N)^{-1} \mathcal{G}^{a b c d} \dot{g}_{a b} \dot{g}_{c d}, \\
& U=U^{\mathrm{G}}+U^{\text {source }}, \\
& U^{\mathrm{G}}=N\left(-g^{1 / 2} R\right), \quad U^{\text {source }}=N\left(-2 g^{1 / 2} k T^{\perp}{ }_{\perp}\right),
\end{aligned}
$$

supplemented by a nonpotential force [6] defined by

$$
-d\left(N^{-1} U\right)+Q^{a b} d g_{a b}=-g^{1 / 2}\left(G^{a b}-k T^{a b}\right) d g_{a b}
$$

for some symmetry types, where the exterior derivative is the one on the space $\mathcal{M}$ of metric matrices, on which the DeWitt [13] metric is a Lorentz metric

$$
2 N^{-1} \mathcal{G}=2 N^{-1} g^{1 / 2}\left(g^{a(c} g^{d) b}-g^{a b} g^{c d}\right) d g_{a b} \otimes d g_{c d} .
$$

Allowing the lapse to have a factor depending explicitly on the spatial metric is equivalent to conformally rescaling this metric by that factor [4]. In particular, the Taub time gauge removes the metric determinant factor and makes $\left\{\alpha, \beta^{A}\right\}$ natural Lorentz orthonormal coordinates on the flat diagonal submanifold $\mathcal{M}_{\mathrm{D}}$ with respect to the rescaled DeWitt metric (a conventional factor of 2 is missing from the kinetic energy)

$$
\left.2 N_{\mathrm{TTG}}^{-1} \mathcal{G}\right|_{\mathcal{M}_{\mathrm{D}}}=-d \alpha \otimes d \alpha+\delta_{A B} d \beta^{A} \otimes d \beta^{B} .
$$

$\alpha$ is the natural time variable on the space, and $\beta^{A}$ are natural spatial coordinates.

If $N$ is considered as an independent variable, one obtains the Einstein evolution equations with the super-Hamiltonian constraint as its Lagrange equation. Allowing the lapse to depend explicitly on the spatial metric leads to new evolution equations which differ by multiples of that constraint. (The Hamiltonian approach widens this freedom to allow explicit dependence on the gravitational momenta.) For diagonal metrics, the useful lapse choices are power law lapses, namely products of powers of the diagonal metric components $[14,15]$. The Taub time gauge choice is an example.

\section{Exact Solutions}

All known exact spatially homogeneous solutions of gravitational field equations are such that the nongravitational variables decouple from the field equations for the gravitational variables in the following sense. The source energy-momentum (including a possible cosmological constant) may be represented in terms of the metric and constants of the motion, leading to an entirely geometric system. 
This occurs only when the source has either discrete or continuous additional symmetry. For the diagonal case, the spatial curvature potential is a linear combination of individual potential terms which are power law in the diagonal metric components and therefore exponentials whose arguments are linear functions of the natural inertial coordinates [6]. To admit exact solutions, the source can add at most more such terms to the total potential energy $U$ when reexpressed in terms of possible constants of the motion. Independent of this, one must have a suitable decoupling of the gravitational modes for exact solutions to exist. At most one power of any exponential argument can appear in the total potential, i.e., the exponentials with different arguments must be associated with linearly independent directions in the space $\mathcal{M}_{\mathrm{D}}$. If this is so, one may obtain integrable decoupled equations, the only complication being the causal nature of the level hypersurfaces (hyperplanes) of the individual exponential potentials. These hyperplanes may be timelike, so that level surfaces move with speed $d s / d \alpha<1$ in $\beta^{A}$ space, or spacelike or null, in the latter case moving with speed $d s / d \alpha=1$. If different powers of a given exponential argument appear, the resulting integrals for decoupled modes become more complicated.

Consider first the four-dimensional vacuum case (with zero cosmological constant). The diagonal exact solutions fall into two overlapping families of solutions which intersect at the well known Kasner family [16]. The first is the family found by Taub [17] in his introduction of the spatially homogeneous cosmological models. He used the time gauge $N=g^{1 / 2}$, namely the Taub time gauge apart from a constant. The second family is due to Joseph [18] and Ellis and MacCallum [19] and contains a subfamily which can be analytically continued to the Kantowski-Sachs models [20]. These latter models are the only nontrivial multiply transitive symmetry type for this dimension in the sense that they admit no subgroup acting simply transitively on the spatial sections and so cannot be represented as a simply transitive case with higher symmetry. The Taub family contains one or two nonnull potentials while the Joseph-Ellis-MacCallum family contains one null potential in Taub time gauge. (Changing the time gauge may change the causality properties.) The Joseph-Ellis-MacCallum solutions have been given in the time gauge $N=\left(g_{33}\right)^{1 / 2}$ but can easily be converted to the Taub time gauge. These two families of exact solutions are very useful examples.

The Taub family of solutions may be described by

$$
\begin{aligned}
& C^{1}{ }_{23}=C^{2}{ }_{31}=n^{(1)}, \quad C^{3}{ }_{12}=n^{(3)}, \quad \mathbf{g}_{\mathrm{D}}=e^{2 \alpha} e^{2\left(\beta^{+} \mathbf{e}_{+}+\beta^{-} \mathbf{e}_{-}\right)}, \\
& \mathbf{e}_{+}=\operatorname{diag}(1,1,-2), \quad \mathbf{e}_{-}=\sqrt{3} \operatorname{diag}(1,-1,0), \quad \beta^{-}=\delta\left(n^{(1)}\right) \beta_{0}^{-},
\end{aligned}
$$

and their dynamics in Taub time gauge is described by the Hamiltonian (expressed in velocity phase space)

$$
H_{\mathrm{TTG}}=\frac{1}{2}\left(-\dot{\alpha}^{2}+\dot{\beta}^{+2}+\dot{\beta}^{-2}\right)+6 n^{(3) 2} e^{4\left(\alpha-2 \beta^{+}\right)}-24 n^{(3)} n^{(1)} e^{-2\left(\beta^{+}-2 \alpha\right)} .
$$

The Lorentz transformation [4]

$$
\left(\bar{\alpha}, \bar{\beta}^{+}\right)=3^{-1 / 2}\left(2 \alpha-\beta^{+},-\alpha+2 \beta^{+}\right)
$$


leads to a completely decoupled Hamiltonian

$$
\begin{aligned}
H_{\mathrm{TTG}} & =\frac{1}{2}\left(-\dot{\bar{\alpha}}^{2}+\dot{\bar{\beta}}^{+2}+\dot{\beta}^{-2}\right)+6 n^{(3) 2} e^{-4 \sqrt{3 \bar{\beta}^{+}}}-24 n^{(3)} n^{(1)} e^{2 \sqrt{3} \bar{\alpha}} \\
& =-H_{\bar{\alpha}}+H_{\bar{\beta}^{+}}+H_{\beta^{-}}=0
\end{aligned}
$$

consisting of three 1-dimensional scattering problems with exponential potentials and constant energies, restricted only by the constraint that the appropriately signed sum of the individual energies vanish. The solution of an exponential scattering problem leads to hyperbolic or trigonometric functions

$$
\begin{gathered}
H_{x}=\frac{1}{2}\left(\dot{x}^{2}+\mu e^{\nu x}\right)=E_{x} \rightarrow t=\int d x\left(2 E_{x}-\mu e^{\nu x}\right)^{-1 / 2}, \\
\text { or defining } \gamma=\left(2 E_{x}\right)^{1 / 2}, \\
e^{-\frac{1}{2} \nu x}=\left\{\begin{array}{lll}
\mu^{1 / 2} \gamma^{-1} \cosh \left(\frac{1}{2} \gamma \nu t\right) & \mu>0, & E_{x}>0 \\
|\mu|^{1 / 2} \gamma^{-1} \sinh \left(\frac{1}{2} \gamma|\nu t|\right) & \mu<0, & E_{x} \in \Re .
\end{array}\right.
\end{gathered}
$$

$\left(\bar{\alpha}, \bar{\beta}^{+}, \beta^{-}\right)$are inertial coordinates of the rest frame of the $n^{(3) 2}$ potential, which moves with speed $d \beta^{+} / d \alpha=\frac{1}{2}$ in $\beta^{A}$ space. For the Bianchi type IX case, characterized by $n^{(3)} n^{(1)}>0$ (so $\beta^{-}=0$ ), the hyperbolic cosine solution is relevant, interpolating between the asymptotic free positive and negative exponential solutions (for the metric components) at $t= \pm \infty$; the unit velocities in $\beta^{A}$ space of the asymptotic solutions are related by a simple reflection in the rest frame of this potential [4]. Letting $n^{(1)} \rightarrow 0$ contracts the group to Bianchi type II, eliminating the "tachyonic" $n^{(3)} n^{(1)}$ potential (it moves with speed $d \beta^{+} / d \alpha=2$ in $\beta^{A}$ space) and allows free motion parallel to the $n^{(3) 2}$ potential (the $\bar{\alpha}$ and $\bar{\beta}^{-}$directions). For the Bianchi type VIII case, characterized by $n^{(3)} n^{(1)}<0$, only the positive energy solutions are relevant for the $\bar{\alpha}$ motion (the potential is negative) due to the super-Hamiltonian constraint. When both $n^{(1)}$ and $n^{(3)}$ vanish, one obtains the Abelian case of Bianchi type I and all three variables have free motion, leading to the Kasner solution when re-expressed in proper time gauge, the exponentials in the Taub time converting to powers of the proper time.

The Joseph-Ellis-MacCallum family is also described by a Hamiltonian system for a diagonal metric matrix but with structure constants and Taub time gauge potential given by

$$
\begin{aligned}
& C^{1}{ }_{31}=a+q, \quad C^{2}{ }_{32}=a-q, a^{2} \equiv-h q^{2}, \quad \lambda \equiv q a^{-1}= \pm(-h)^{-1 / 2}, \\
& U_{\mathrm{TTG}}=12 \cdot 6 e^{4\left(\beta^{0}+\beta^{+}\right)}\left(a^{2}+q^{2} / 3\right)=12 \cdot 6 a^{2} e^{4\left(\beta^{0}+\beta^{+}\right)}\left(1+\lambda^{2} / 3\right) .
\end{aligned}
$$

The null hyperplane level surfaces of this potential require a different transformation of coordinates when $a \neq 0$

$$
\begin{array}{llrl}
\bar{\beta}^{0} & =\beta^{0}+\beta^{+} & \beta^{0} & =\left(1+\zeta^{2} / 3\right) \bar{\beta}^{0}-\bar{\beta}^{+}+\zeta \bar{\beta}^{-} / \sqrt{3} \\
\bar{\beta}^{+} & =\beta^{+}+\zeta \beta^{-} / \sqrt{3} & \beta^{+} & =\bar{\beta}^{+}-\zeta \bar{\beta}^{-} / \sqrt{3}-\zeta^{2} \bar{\beta}^{0} / 3 \\
\bar{\beta}^{-} & =\beta^{-}-\zeta\left(\beta^{0}+\beta^{+}\right) / \sqrt{3} & \beta^{-} & =\bar{\beta}^{-}+\zeta \bar{\beta}^{0} / \sqrt{3},
\end{array}
$$


where $\zeta \equiv[1-\delta(a)] \lambda$, leading to

$$
\begin{aligned}
& H_{\mathrm{TTG}}=-\frac{1}{2}\left(1+\zeta^{2} / 3\right)\left(\bar{\beta}^{0}\right)^{\cdot 2}+\frac{1}{2}\left(\bar{\beta}^{0}\right)^{\cdot}\left(\bar{\beta}^{+}\right)^{\cdot}+\frac{1}{2}\left(\bar{\beta}^{-}\right)^{\cdot 2}+12 \cdot 6 a^{2} e^{4 \bar{\beta}^{0}}\left(1+\lambda^{2} / 3\right) \\
& \mathcal{H}^{\perp}{ }_{3}=-12 a e^{\bar{\beta}^{0}}\left(\bar{\beta}^{+}\right) \quad\left[\text { note: } a \bar{\beta}^{+}=a \beta^{+}+q \beta^{-} / \sqrt{3}\right] .
\end{aligned}
$$

If $a \neq 0$, then $\left(\bar{\beta}^{+}\right)^{\cdot}=0$ (set its constant value equal to zero since it is gauge) and the Hamiltonian becomes

$$
H_{\mathrm{TTG}}=-\left(1+\lambda^{2} / 3\right) H_{\bar{\beta}^{0}}+H_{\bar{\beta}^{-}},
$$

thus decoupling into free motion for $\bar{\beta}^{-}$with constant energy (linear behavior) and a one-dimensional scattering problem for $\bar{\beta}^{0}$ in a negative exponential potential with constant energy (the "ln sinh" solution) which must be positive to satisfy the vacuum super-Hamiltonian constraint of vanishing total energy.

If $a=0$ and therefore $\zeta=0$, then $\beta^{+}$and $\beta^{-}$are unchanged by the transformation while $\bar{\beta}^{0}=\beta^{0}+\beta^{+}$and it is convenient to introduce another null coordinate $\bar{\beta}^{+} \equiv \beta^{+}-\beta^{0}$ so that the kinetic energy becomes

$$
T_{\mathrm{TTG}}=\frac{1}{2}\left(\bar{\beta}^{0}\right)^{\cdot}\left(\bar{\beta}^{+}\right)^{\cdot}+\frac{1}{2}\left(\beta^{-}\right)^{\cdot 2} .
$$

Here the null geometry is essential. $\beta^{-}$and $\bar{\beta}^{0}$ undergo free motion while $\left(\bar{\beta}^{+}\right)^{*}=8 U_{\text {TTG }}$ leading to exponential behavior, describing the Bianchi type $\mathrm{VI}_{0}$. However, if $q \neq 0$, then the supermomentum constraint requires that $\beta^{-}$ be a constant (set it equal to zero since it is gauge) while if $q=0$ one arrives again at the Abelian case and all three variables are free. The Bianchi type $\mathrm{VI}_{0}$ solution in proper time time gauge was given by Ellis and MacCallum [19].

The condition $a=q \neq 0$ (or $a=-q$ ) describes Bianchi type III $=\mathrm{VI}_{-1}$. The vacuum supermomentum constraint then imposes the condition $\left(g_{11} / g_{33}\right)^{\circ}=0$ associated with local rotational symmetry, the spatial geometry being that of a family of geodesically parallel 2-dimensional surfaces of constant negative curvature. Allowing $q^{2}$ to take negative values in the Einstein equations simply changes the sign of the potential and describes the Kantowski-Sachs models which instead have constant positive curvature on the geodesically parallel family of 2-dimensional surfaces, changing the solutions of the one-dimensional scattering problem from hyperbolic to trigonometric functions.

All of these solutions describe the effect of zero, one or two independent exponential potentials on the flat diagonal configuration space $\mathcal{M}_{\mathrm{D}}$. They can be extended to certain nonvacuum nonzero cosmological constant cases in four and higher dimensions in a trivial way by a mechanism called "variation of parameters" [15]. The constraints impose certain values on the parameters which appear in the evolution equations. Allowing the parameters to vary from their constrained values is equivalent to introducing a nonzero energy-momentum tensor which may be interpreted as that of a stiff perfect fluid moving orthogonal to the spatial sections if the supermomentum is zero, or equivalently as a massless scalar field. Nonzero values of the supermomentum in the Joseph-EllisMacCallum case lead to tilted fluid flow and can also be interpreted in terms 
of changing the spacetime symmetry from isometries to homothetic transformations, leading to spatially self-similar solutions.

The massless scalar field can be obtained by dimensional reduction from a 5-dimensional vacuum Kaluza-Klein theory, together with a Weyl transformation, namely a conformal transformation of the four-dimensional metric when imbedded in five dimensions [21]. This allows one to relate the above solutions to solutions of the Brans-Dicke theory [21-23]. One may also extend the imbedding to vacuum Kaluza-Klein theories with any number of additional flat dimensions, thus obtaining exact solutions of those theories $[7,24]$. The Weyl transformation is related to a redefinition of the variable $\alpha$ when adding extra dimensions.

Nonvacuum Kaluza-Klein theories also admit such an extension of the fourdimensional vacuum solutions by variation of parameters. Both ten and elevendimensional supergravity when restricted to the Bose sector merely add additional exponential potentials to the spatial curvature potentials when one assumes a Freund-Rubin [25] or Freund-Rubin-Englert [26] ansatz, since the differential form fields can then be expressed in terms of the metric and constants of the motion. The scalar field in the ten-dimensional case is related by dimensional reduction to an extra flat dimension [27]. One thus sees the same familiar exact solutions reappearing [28-31].

These solutions may also be deformed by a second kind of variation of parameters in which one changes the parameters which appear in the field equations themselves in such a way that the equations still remain integrable. In the Abelian case, the addition of a cosmological constant or a perfect fluid with equation of state $p=(\gamma-1) \rho$ whose flow is orthogonal to the spatial sections adds a single $\alpha$-dependent exponential to the Hamiltonian, a timelike potential like one of the two in the semisimple Taub case in four dimensions. One can add such a potential to the nonsemisimple Taub case or the Joseph-Ellis-MacCallum case and still obtain the same kinds of solutions. On the other hand, adding a locally rotationally symmetric electromagnetic field changes the nature of the solutions, but only deforms them in a simple way, leading to more very similar exact solutions. One may in fact add many fields simultaneously, leading to, for example, solutions in four dimensions with a stiff perfect fluid, a scalar field and an electromagnetic field, the scalar field allowing the optional reinterpretation in terms of solutions of the Brans-Dicke theory.

Consider adding a locally rotational electromagnetic field to the Taub family of models in four dimensions [32]. This simply adds another $\bar{\beta}^{+}$potential to the Hamiltonian but with a different power, leading to a one-dimensional scattering problem with two different exponential potentials. The explicit integral in (17), when rewritten in terms of the variable $u=\mu^{-1 / 2} e^{-\frac{1}{2} \mu x}$, becomes proportional to the standard integral $\left(\int d u\left(c^{2} u^{2}-1\right)^{-1 / 2}\right)$ for the inverse hyperbolic cosine when $\mu$ and $E_{x}$ are positive as occurs in this case. The additional potential merely adds a linear term to the radical which can be eliminated by completing the square. The new solution for $u$ is then a constant plus another constant times the old solution, a simple variation of parameters [15,33]. 
The Taub time gauge is clearly very powerful, but other power law lapse time gauges are also useful. Changing the lapse leads to different decoupling possibilities for different kinds of driving terms. For example, adding a cosmological constant to the semisimple Taub case (Bianchi types VIII and IX) leads to three exponential potentials along distinct directions in the two-dimensional Taublike subspace of $\mathcal{M}_{\mathrm{D}}$ associated with local rotational symmetry. In addition to the orthogonal pair of timelike and spacelike potentials, a cosmological constant term adds a null potential, causing a coupling of the equations. However, in Misner's [15,34] time gauge $N \sim\left(g_{33}\right)^{-1 / 2}$, one degree of freedom remains unchanged but enters the equation of motion for the second in such a simple way that the new solution merely adds on a term to the old one [35]. There are many other instances in various time gauges where similar ideas apply (including the Joseph-Ellis-MacCallum family in the original time gauge and even the familiar Friedmann models in conformal time gauge $N \sim e^{\alpha}$, proper time time gauge and other time gauges, as well as higher dimensional generalizations of these solutions). However, no systematic analysis of lapse choices has ever been undertaken as a way of simplifying the equations of motion, other than the limited discussions of references [7] and [15].

It is incredible how many pages of journal space have regurgitated the same old solutions in various disguised forms without leading to some recognition of the connections. Too many people interested in exact cosmological solutions suffer to some degree from a sort of tunnel vision which prevents them from taking the time to appreciate the ADM perspective for the very branch of gravitation for which it is the most powerful. The present article shows that the rewards for doing this are considerable.

\section{Acknowledgment}

J. Demaret is thanked for providing the stimulus to consider these questions and L. Angelini for helpful discussion during the course of their development.

\section{References}

[1] R. Arnowit, S. Deser and C.W. Misner, The Dynamics of General Relativity, in: Gravitation: An Introduction to Current Research, ed. L. Witten (Wiley, New York, 1962).

[2] C.W. Misner, Phys. Rev. 186 (1969) 1319.

[3] C.W. Misner, Phys. Rev. Lett. 22 (1969) 1074.

[4] C.W. Misner, Classical and Quantum Dynamics of a Closed Universe, in: Relativity, eds. M. Carmeli, S.I. Fickler and L. Witten (Plenum Press, New York, 1970); Minisuperspace, in: Magic Without Magic, ed. J.R. Klauder (Freeman, San Francisco, 1972). 
[5] M. Henneaux, Phys. Lett. 168B (1986) 233.

[6] R. T. Jantzen, Phys. Rev. D34 (1986) 424.

[7] R. T. Jantzen, Higher Dimensional Cosmological Models: The View From Above, in: Proc. 26th Liège Int. Astrophys. Colloq., ed. J. Demaret (Liège University Press, Liège, 1986).

[8] L. Castellani, L.J. Romans and N.D. Warner, Ann. Phys. (N.Y.) 157 (1984) 394.

[9] L. Castellani, Phys. Lett. 149B (1984) 103.

[10] R.T. Jantzen, Ann. Phys. (N.Y.) 145 (1983) 378; Phys. Rev. D33 (1986) 2121.

[11] R.T. Jantzen, Spatially Homogeneous Dynamics: A Unified Picture, in: Cosmology of the Early Universe, eds. R. Ruffini and Fang L.Z. (World Scientific, Singapore, 1984) and in: Gamov Cosmology, eds. R. Ruffini and F. Melchiorri (North Holland, Amsterdam, 1987).

[12] C.W. Misner, J.A. Wheeler and K.S. Thorne, Gravitation (Freeman, San Francisco, 1973).

[13] B.S. DeWitt, Phys. Rev. 160 (1967) 1113.

[14] S. Bonanos, Commun. Math. Phys. 22 (1971) 190.

[15] R.T. Jantzen, Ann. Phys. (N.Y.) 127 (1980) 302.

[16] D. Kramer, H. Stephani, M. MacCallum and E. Herlt, Exact Solutions of Einstein's Field Equations (Cambridge University Press, Cambridge, 1980).

[17] A.H. Taub, Ann. Math. 53 (1951) 472.

[18] V. Joseph, Proc. Camb. Phi. Soc. 62 (1966) 87.

[19] G.F.R. Ellis and M.A.H. MacCallum, Commun. Math. Phys. 12, (1969) 108.

[20] R. Kantowski and R.K. Sachs, J. Math. Phys. 7 (1968) 443.

[21] V.A. Belinsky and I.M. Khalatnikov, Sov. Phys. JETP 36 (1973) 591.

[22] E.R. Harrison, Phys. Rev. D6 (1972) 2077.

[23] D. Lorentz-Petzold, Exact Brans-Dicke-Bianchi Solutions, in: Lecture Notes in Physics 205, eds. C. Hoenselaers and W. Dietz (Springer, Berlin, 1984).

[24] J.D. Barrow and J. Stein-Schabes, Phys. Rev. D32 (1985) 1595.

[25] P.G.O. Freund and M.A. Rubin, Phys. Lett. 97B (1980) 233. 
[26] F. Englert, Phys. Lett. 119B (1982) 339.

[27] P.G.O. Freund, Nuc. Phys. B209 (1982) 146.

[28] D. Lorentz-Petzold, Phys. Lett. 148B 43 (1984), 149B 79 (1984), 151B 105 (1985), 158B 110 (1985), 167B 157 (1986); Prog. Theor. Phys. 73533 (1985); Class. Quantum Grav. 2829 (1985).

[29] D. Lorentz-Petzold, Phys. Lett. 175B (1986) 405.

[30] J. Demaret, J.-L. Hanquin, M. Henneaux and P. Spindel, Nuc. Phys. 252 (1985) 538.

[31] J. Demaret and J.-L. Hanquin, Phys. Rev. D31 (1985) 258.

[32] D. Brill, Phys. Rev. 133B (1964) 845.

[33] M.A.H. MacCallum, Gen. Relativ. Grav. 17 (1985) 659.

[34] C.W. Misner and A.H. Taub, Sov. Phys. JETP 28 (1969) 122.

[35] D. Brill and F. Flaherty, Ann. Inst. H. Poincaré A28 (1978) 335. 